\section{Birth defects}

\section{Are offspring at risk from their father's exposure to toxins?}

\section{from Nigel A. Brown}

Congenital malformations can be induced by exposure of the mother to teratogens in early pregnancy, but can they also be caused by treatment of the father before, even long before, conception? The answer, as far as we know it, draws on observations of the effects of genetic lesions, indirectly addressed on page 144 of this issue ${ }^{1}$, and on a consideration of potential epigenetic mechanisms.

The various stages of male germ-cell maturation can be differentially susceptible to toxic insult ${ }^{2}$. It is simplest to distinguish two overall phases - pre-meiotic and post-meiotic. The pre-meiotic stem cells produce spermatogonia for the whole reproductive lifespan and they cannot be replaced if destroyed. The lifespans of post-meiotic stages are short by comparison and damaged cells will disappear within one cycle of spermatogenesis, a matter of a few weeks, whereas an abnormal stem cell may be retained for life.

It is possible for treatment of a male to induce genetic damage in germ cells which causes congenital malformation in his offspring. Male mice exposed to X rays or to the mutagen urethane produce fetuses with an increased incidence of malformations $s^{3,4}$. Various common defects are observed, some that would be lethal after birth, others that seem just to be a reduction in fetal size. With postmeiotic exposure of germ cells, up to 4.5 per cent of fetuses are defective, whereas pre-meiotic treatment produces a lower, but still significant, incidence of defects. The malformations are probably caused by both small chromosomal aberrations and gene mutations, and are obviously genetically dominant ${ }^{4}$. Congential cataracts and skeletal defects have also been observed in offspring sired many months after male mice have been exposed to $X$ rays or ethylnitrosourea ${ }^{s-7}$. Breeding of these defective offspring has shown (with some technical reservations ${ }^{8}$ ) that the malformations can be inherited and so are clearly caused by dominant mutations, originally induced in spermatogonia.

Not all embryos produced by mutant spermatozoa result in malformed fetuses. Many embryos die, usually shortly after implantation, due to major aberrations in chromosome numbers or structure induced in post-meiotic stages. Structurally normal offspring can be born with phenotypic changes, ranging from minor coat colour or behavioural abnormalities to potentially lethal biochemical or functional deficits. Limited information suggests that the incidence of cancer in mice can be increased by paternal treatment with mutagens ${ }^{3,9}$.

Trasler et al. ${ }^{1}$ describe birth defects produced by treating male rats with cyclophosphamide, a mutagenic drug widely used in cancer chemotherapy. They confirm that chronic low doses of cyclophosphamide produce many embryonic deaths and report a significant incidence of up to seven per cent malformed fetuses, in contrast to a previous study using a higher dose where only behavioural anomalies in offspring were observed ${ }^{10}$. A preliminary report of acute high-dose exposure has also suggested a weak increase in malformations ${ }^{11}$. The current study uses small groups of treated males, so the number of abnormal fetuses is low and could be due to chance distribution of spontaneous defects, particularly as outbred rats are used. If the malformations are a genuine result of treatment, the observation is significant - many humans are exposed to equivalent doses and there is a suggestion that pre-meiotic germ cells are involved.

Epigenetic mechanisms may also be involved, as some chemicals not usually thought to be genotoxic seem also to affect offspring following paternal treatment. In rodents, paternal exposure to ehtanol ${ }^{\mathrm{t} 2}$ or narcotics ${ }^{13}$ can lead to reductions in litter size and birthweight, neonatal survival and behavioural deficiencies, but no reproducible increase in malformations. Ethanol may have some genetic effects ${ }^{14.15}$ but it is far from a potent mutagen.

There is little mechanistic evidence to support paternally-induced birth defects by epigenetic events. It seems unlikely that damage to non-genetic components of sperm results in malformations, because recent micromanipulation experiments suggest that sperm cytoplasm is not essential for normal embryogenesis ${ }^{16}$. Chemicals can be transported from male to female in seminal fluid or bound to spermatozoa ${ }^{17}$ but no fetal malformations have been observed that can be attributed to treatment of gametes at fertilization or of embryos at pre-implantation stages. It seems unlikely that a chemical could retain its activity in the uterus until the embryo reaches the teratogen-sensitive stage of organogenesis.

In humans, epidemiological data suggest that certain occupations increase the risk of fathering an abnormal child ${ }^{18}$, but such data are notoriously susceptible to bias from ethnic and socio-economic factors. Two groups of men have been exposed to high levels of genotoxic agents: survivors of the atom bomb explosions and of cancer therapy. There is no evidence of increased incidence of birth defects in children of people exposed to atomic radiation ${ }^{19}$. Men in long-term remission from cancer are less well studied but the current impression is that there is no increased risk of malformation among their children, although this is based on clinical observations with limited power to detect even a modest increase in risk. Because these men represent a unique opportunity to evaluate the possible induction of transmissible genetic damage in humans, efforts should be made to study them carefully.

There has been concern over human exposure to 'Agent Orange' although neither of its components, 2,4-D and $2,4,5-\mathrm{T}$, or the contaminant dioxin are potent mutagens. No defects have been observed in the offspring of male animals experimentally exposed to dioxin, even though it is an effective teratogen ${ }^{20}$. Some studies of children of men exposed to Agent Orange have claimed that birth defects are increased but most have not, and none is large or complete enough to be definitive. Several large studies in progress, particularly of Vietnam veterans, should provide more convincing answers ${ }^{21}$

It would be foolish to advise anything other than extreme caution over the exposure of humans to chemical mutagens when our understanding of the quantitative risks to future generations is so rudimentary. Many fear that the human mutational load is being increased by chemically induced recessive changes which are currently un-noticed. As far as human birth defects are concerned, the limited data are generally comforting but this is not an excuse for complacent inactivity. While we must be thankful that no known chemical induces birth defects by an epigenetic mechanism following paternal exposure, it has to be admitted that such an agent would be an intriguing tool for reproductive toxicologists.

\footnotetext{
1. Trasler, J. M., Hales, B. F. \& Robaire, B. Nature 316, 144 (1985)

2. Lyon, M. F. Mutat. Res.87, 323 (1981)

3. Nomura, T. Nature 296, 575 (1982)

Kirk, K. M. \& Lyon, M. F. Mutat. Res, 125, 75 (1984)

5. Selby, P. B. \& Selby, P. R. Mutat. Res, 43, 357 (1977).

6. Kratochvilova, J. J. Hered. 72, 302 (1981).

. Favor, J. Genet. Res. 44, 183 (1984).

Favor, J. Genet. Res. 44, $183(1984)$.
West, J.D. et al. Genet, Res. 44, 343 (1984)

Tomatis, L. el al. Int. J. Cancer 28, $475(1981)$

10. Adams, P. M. et al. Science 211, 80 (1980)

11. Knudsen, I. et al. Mutat. Res. 48, 267 (1977).

12. Anderson, R. A. in Fetal Alcohol Syndrome Vol. 3 (ed. Abel, E. L.) 83 (CRC, Boca Raton, 1982)

13. Joffe, J. M. Clin. Perinotol. 6, 21 (1979).

14. Kaufman, M. H. Nature 302, 259 (1983).

15. Badr, F. M. \& Badr, R. S. Nature 253, 134 (1975).

16. Surani, M. A. H. et al. Nature 308,548 (1984)

17. Mann, T. \& Lutwak-Mann, C. Crit. Rev. Tox. 11, 1 (1983).

18. OPCS Monitor MD 382/1, UK Government Statistical Service (1982)

19. Miller, R. W. Pediatrics 41, 257 (1968)

20. Pearn. J. H. Med. J. Aust. 2, 16(1983)

21. Budiansky. S. Nature 308, 102 (1984).
}

Nigel A. Brown is in the Medical Research Council Experimental Embryology and Teratology Unit, Carshalton, Surrey SM5 4EF, UK. 